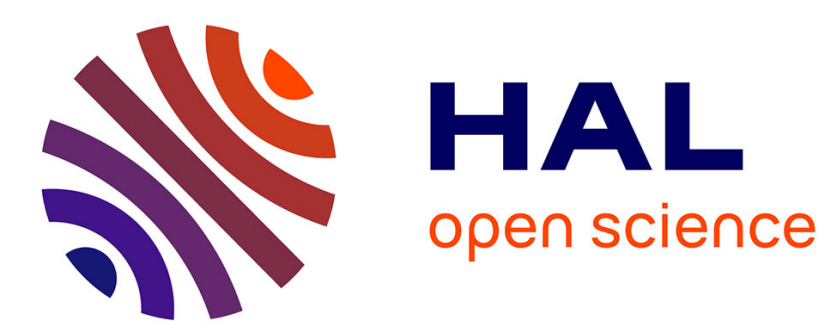

\title{
Sur les raies spectrales spontanément renversables et l'analogie de leurs lois de répartition et d'intensité avec celles des raies de l'hydrogène
}

\author{
A. Cornu
}

\section{- To cite this version:}

A. Cornu. Sur les raies spectrales spontanément renversables et l'analogie de leurs lois de répartition et d'intensité avec celles des raies de l'hydrogène. J. Phys. Theor. Appl., 1886, 5 (1), pp.93-100. 10.1051/jphystap:01886005009300 . jpa-00238700

HAL Id: jpa-00238700 https://hal.science/jpa-00238700

Submitted on 1 Jan 1886

HAL is a multi-disciplinary open access archive for the deposit and dissemination of scientific research documents, whether they are published or not. The documents may come from teaching and research institutions in France or abroad, or from public or private research centers.
L'archive ouverte pluridisciplinaire HAL, est destinée au dépôt et à la diffusion de documents scientifiques de niveau recherche, publiés ou non, émanant des établissements d'enseignement et de recherche français ou étrangers, des laboratoires publics ou privés. 


\section{SUR LES RAIES SPECTRALES SPONTANÉMENT RENVERSABLES ET L'ANA- LOGIE DE LEURS LOIS DE RÉPARTITION ET D'INTENSITÉ AVEG GELLES DES RAIES DE L'HYDROGENE;}

Par M. a. CORNU.

Le progrès des études spectrales et l'extension merveilleuse de leurs applications ont posć l'un des plus beaux problèmes qui se soient jamais offerts aux expérimentateurs et aux théoriciens :

Étant donnée la composition chimique d'une vapeur portée à l'incandescence dans des conditions définies, prévoir la répartition des raies du spectre des radiations émises et leur intensité relative.

Quoique l'attention des observateurs ait été bien des fois attirée sur ce problème, la solution n'en paraît pas très avancée : je me propose, après avoir rappelé brièvement les points sur lesquels les efforts sont venus échouer, d'indiquer un ordre de faits qui semblent ouvrir une voie nouvelle.

Le point de départ de toutes les recherches de ce genre est la remarque suivante :

Lorsqu'on examine les spectres lumineux des vapeurs incandescentes, on distingue souvent des groupes de raies qui se reproduisent avec une périodicité plus ou moins régulière : ces répétitions sont encore plus frappantes lorsqu'on étend le champ des observations jusqu'à la limite extrême du spectre ultra-violet.

L'analogie de ces groupes périodiques avec les harmoniques des corps sonores se présente immédiatement à l'esprit. L'idée est d'autant plus séduisante que les positions de ces groupes, considérées tantôt sur l'échelle des longueurs d'onde, tantôt sur celle des nombres de vibrations, paraissent coüncider avec des termes successifs de la série des nombres entiers. Aussi n'est-il guère de spectroscopistes qui n'aient pas cédé à cet entraînement et qui n'aient pas cherché à ranger des raies d'un même spectre suivant une série harmonique.

Malheureusement la simplicité des relations numériques s'évanouit toujours, lorsqu'on substitue à la contemplation des images J. de Phys., $2^{\text {e }}$ série, t. V. (Mars 1886 .) 
spcctrales la détermination numérique précise des longueurs d'onde : la voie des spéculations théoriques semble donc fermée de ce côté.

Cet insuccès ne doit pas décourager les observateurs; toutefois l'espoir de trouver une loi simple, comme celle des harmoniques musicaux, témoigne d'une idée préconçue qu'il importe d'écarter immédiatement; cette loi des nombres entiers ne s'applique qu'à une forme très particulière de corps sonores dont le type est la colonne cylindrique de longueur très grande par rapport à la section : si la forme du corps vibrant s'écarte de ce type spécial, la relation entre les nombres de vibrations des sons successifs devient très complexe $\left({ }^{1}\right)$.

Cette simple remarque suffit à montrer qu'il serait puéril de chercher comme loi générale une relation aussi simple que celle des harmoniques des instruments musicaux : ce serait supposer à la structure des molécules incandescentes une constitution mécanique que l'ensemble des phénomènes chimiques ou physiques ne pourrait guère justifier. Des lois moins simples ne réussissent pas mieux et cela ne doit point étonner : les considérations précédentes montrent même qu'on doit s'attendre à rencontrer, pour définir la répartition des raies spectrales, des fonctions très complexes dépendant de la nature chimique de l'élément, de la structure moléculaire de la vapeur, des conditions d'excitation vibratoire, etc.

En fait, ce qui paraît avoir rendu stériles les efforts tentés en vue de la solution du problème, c'est qu'on a cru devoir s'imposer

( ${ }^{1}$ Par exemple, dès que les colonnes cylindrıques présentent une section comparable à leur longueur, ce qui est le cas des tiges ou des verges, le nombre $n$ des vibrations transversales est donné, suivant que les extrémités sont fixes ou libres, par les racincs de l'équation transcendante

$$
\frac{e^{+h \sqrt{n}}-e^{-h \sqrt{n}}}{2} \cos k \sqrt{n} \pm \mathrm{I}=0
$$

l'intensité de chaque son dépend, d'äilleurs, des fonctions qui définissent les conditions initiales du mouvement. Les valeurs de ces racines, qui ne sont pas sans analogie avec les intervalles de certaines raies spectrales, approchent indéfiniment de la série plus simple $k \sqrt{n}=(2 i \pm \mathrm{r}) \frac{\pi}{2}$ lorsque l'ordre $i$ grandit suffisamment. 
a priori la fonclion destinée à représenter la succession des raies spectrales. Je crois qu'il faut au contraire, rejetant toute idée préconçue sur l'expression mathématique du phénomène, chercher par expérience s'il n'existe pas de fonctions spéciales (exprimables ou non par les symboles ordinaires de l'analyse) susceptibles de réunir dans une loi commune ces séries, dont la régularité et l'analogie ne sont évidemment pas fortuites.

Envisagée à ce point de vue, la question change donc de face et le problème consiste à rechercher si dans tous les spectres il ne se rencontre pas quelques groupes de raies présentant des caractères communs, indépendants de la nature chimique de la vapeur incandescente.

C'est dans cet ordre d'idées que depuis longtemps je poursuis l'étude des spectres visibles et ultra-violets, et je suis parvenu à reconnaître qu'il existe effectivement une catégorie de raies spectrales offrant des caractères si distincts qu'on ne saurait les confondre avec les autres : ce sont les raies spontanément renversables que j'ai décrites en 187 I (1) et dont j'ai montré l'existence dans la plupart des spectres de vapeurs métalliques. La disposition commune de leurs groupements, comme on le verra bientôt, me paraît définir une de ces formes expérimentales de fonction répondant aux conditions énoncées. Voici d'ailleurs l'exposé des faits.

Caractères des raies spontanément renversables. - Ils sont bien connus des physiciens et ont été observés, pour la première fois, dans la combustion du sodium par M. Fizeau; je les rappellerai brièvement. Une vapeur incandescente, sous faible densité et à basse température, émet une certaine radiation qui correspond, dans l'observation spectrale, à une certaine raie brillante, mais très fine; si l'on augmente progressivement la température et la densité de la vapeur, la raie croît en intensité et en largeur et devient une véritable bande lumineuse à bords estompés : bientôt on voit, sur cette bande, naître une ligne sombre à la place de la raie fine primitive; finalement, la bande lumineuse s'élargit d'une manière en quelque sorte indéfinie, offrant toujours la ligne

(1) Comptes rendus, t. LXXIII, p. 332. 
sombre qui s'élargit aussi d'une manière à peu près proportionnelle. Sur le fond lumineux produit par l'épanouissement de la bande, les raies brillantes non renversables s'évanouissent complètement.

Ces phénomènes, observables dans le spectre visible avec la plupart des métaux volatils et l'étincelle d'induction, présentent des proportions énormes dans l'arc électrique, et surtout dans la région ultra-violette. Je citerai en particulier la raie $\lambda=\mathbf{2 2} 8,85$ du cadmium; extrêmement faible avec l'étincelle d'induction, cette raie s'élargit dans l'arc au point d'envahir la presque totalité du spectre photographique; quant à la bande sombre de renversement qui en forme le centre, elle arrive à occuper plus de la moitié de l'intervalle compris entre les raies 23 et $\mathbf{2 4}$ (notations de M. Mascart), qui disparaissent presque complètement. On peut encore citer la raie $\lambda=209 \mathrm{du}$ zinc; deux des composantes de la raie quadruple du magnésium, $\lambda=280$, ainsi que les belles séries de l'aluminium et du thallium figurées plus loin.

Divers observateurs, en première ligne MM. Liveing et Dewar, ont obtenu un très grand nombre de renversements curieux dans les circonstances les plus diverses. Les plus intéressants sont ceux des raies de l'hydrogène dans le spectre visible, et dans l'ultraviolet celui de la raie $\lambda=285$ du magnésium, qu'on reproduit avec facilité en brûlant le métal à l'air.

J'ajouterai que les raies longues, définies un peu arbitrairement par certains spectroscopistes, rentrent souvent dans la catégorie des raies spontanément renversables.

Il est à peine besoin de rappeler que les lois ordinaires de l'absorption lumineuse et du refroidissement suffisent à expliquer en détail tous ces phénomènes; mais il n'est pas inutile de remarquer que si la théorie prévoit la possibilité du renversement d'une raie donnée, elle ne nous apprend rien sur la propriété de certaines radiations d'offrir, dans des conditions données, le renversement spectral à l'exclusion des autres; c'est cette propriété qui me paraît constituer un élément nouveau et caràctéristique.

Quelles sont en général les conditions de température, de pression et de densité les plus favorables à la production de ce pouvoir émissif singulier? C'est ce qu'il serait difficile de dire actuellement. On l'obtient par l'incandescence des vapeurs métalliques 
dans un espace plus ou moins limité, échauffé par l'arc électrique ou la combustion directe; mais il est évident que ces conditions auraient besoin d'être précisées et qu'elles sont plus ou moins imparfaitement remplies par les divers moyens à l'aide desquels on obtient les spectres d'émission. Quoi qu'il en soit de l'imperfection de ces moyens, l'importance de cette constitution limite s'accroît encore par les rapprochements qui vont suivre.

Disposition particulière des groupes spontanément renversables. - Lorsqu'on examine sur divers clichés photographiques (1) des groupes de raies qui se reproduisent périodiquement avec une régularité particulière, on reconnaît le plus souvent que ces groupes appartiennent précisément à la catégorie des raies spontanément renversables, car les unes sont renversées, les autres sur le point de l'être : pour un même métal, les renversements sont plus ou moins complets suivant les conditions de l'expérience, et pour des métaux différents, suivant les propriétés chimiques et physiques du métal. De plus, la loi de distribution de ces groupes présente un autre caractère commun relativement à la succession des distances et des intensités : les raies vont en se resserrant vers le côté le plus réfrangible et en diminuant d'intensité. Ce caractère est d'autant plus frappant que le nombre des raies renversées est plus considérable, et que le champ sur lequel elles se détachent est plus uniforme; il semble qu'avec l'élévation de température le spectre tende vers un aspect limite, celui d'un fond brillant continu, dépouillé de toute raie autre que celle de la série régulière des raies spontanément renversées. C'est sur cette constitution, en quelque sorte limite, que je désire attirer l'attention des observateurs.

Le nombre des spectres métalliques pouvant donner une série

( $\left.{ }^{1}\right)$ Les clichés photographiques ont l'avantage d'offrir une vue d'ensemble: avec l'appareil dont je me sers (demi-prismes de quartz gauche et droit, lentilles achromatiques quartz fluorine), on obtient sur la même épreuve et avec une netteté satisfaisante la totalité du spectre violct et ultra-violet : la réduction à l'échelle des longueurs d'onde s'effectue à l'aide d'un spectre de comparaison formé des raies du cadmium, du zinc et de l'aluminium dont j'ai déterminé avec soin les longueurs d'onde (Journal de Physique, I $^{\text {re }}$ série, t. X, p. 425); on obtient ce spectre auxiliaire à l'aide d'une étincelle d'induction jaillissant entre deux pointes d'un alliage formé de poids égaux de ces trois métaux. 
régulière de raies spontanément renversées sur un fond continu est assez considérable; mais les plus belles séries que j'aie observées ont été fournies par deux métaux qu'on ne s'attendrait guère,
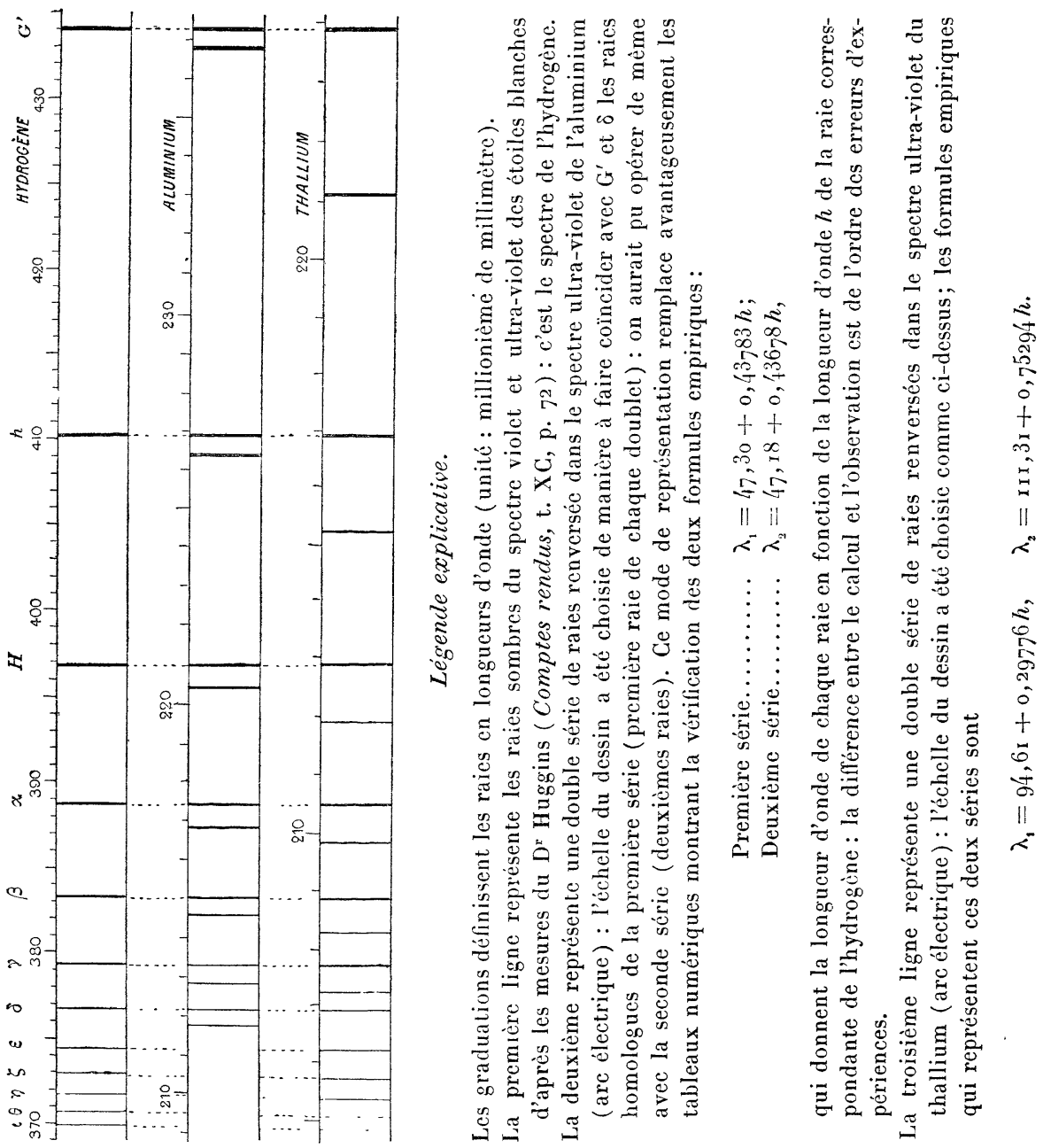

au point de vue chimique, à trouver côte à côte : ce sont l'aluminium et le thallium, dont les équivalents sont aux extrémités de la liste de ceux des corps simples. La figure ci-dessus donne une idée de la répartition de ces raies renversées : on voit qu'elles forment 
dans chaque spectre une série de doublets remplissant les conditions de distance et d'intensité énoncées plus haut.

Analogie avec la répartition des raies de l'hydrogène. - Je ne m'arrêterai pas à indiquer les essais infructueux de calculs numériques que j'avais entrepris en vue de représenter chacune de ces séries par la substitution de la série des nombres entiers dans une fonction simple : je dirai seulement que j'avais abandonné ces recherches lorsque la belle découverte du $D^{r}$ Huggins, sur le spectre des étoiles blanches, ramena vivement mon attention sur ce sujet et m'ouvrit un horizon nouveau. Ces spectres présentent, en effet, une série commune de raies sombres, c'est-à-dire renversées, remplissant précisément les conditions de distance et d'intensité qui caractérisent dans les spectres métalliques les raies spontanément renversables : elle prolonge la série des raies bien connues du spectre de l'hydrogène $\mathrm{C}, \mathrm{F}, \mathrm{G}^{\prime}, h$; on pouvait donc prévoir que la série entière en faisait partie; c'est ce qu'ont depuis confirmé les travaux de $M$. Vogel sans cependant lever toute incertitude. L'intérêt de cette identification était tel que j'ai tenu à la vérifier moi-même, ce que je n'ai pu réaliser que dernièrement ( ${ }^{1}$ ). L'expérience n'est pas sans difficulté; mais, en prenant des précautions de plus en plus minutieuses pour écarter les impuretés du gaz hydrogène raréfié, j’ai vu successivement s'effacer les raies étrangères, et finalemenı j’ai réussi à obtenir des clichés photographiques offrant la série des raies stellaires dans toute leur pureté. (Voir l'article ci-après).

Le spectre de l'hydrogène est placé sur la première ligne du dessin ci-joint : on a rendu la comparaison plus facile par le choix des échelles, de manière à montrer intuitivement l'identité de la loi de répartilion des raies dans les trois spectres. On comparerait de même des groupes plus complexes. comme ceux du magnésium, du zinc, du sodium, etc.; la seule difficulté est d'établir la concordance des groupes; on y arrive immédiatement par une construction graphique assez simple. On en conclut l'énoncé suivant qui résume l'ensemble de mes recherches: Dans les spectres métal-

( ${ }^{1}$ Avec l'obligeant concours de M. Alvergniat. 
liques, certaines séries de raies spontanément renversables présentent sensiblement les mêmes lois de répartition et d'intensité que les raies de l'hydrogène (1).

Il n'est pas nécessaire d'insister longuement sur l'importance de cette relation : elle met en évidence l'existence d'une loi très générale relative aux pouvoirs émissifs des vapeurs incandescentes et, d'autre parı, elle montre que cette loi de succession des raies spectrales, commune à tant de séries, paraît devoir ètre exprimable à l'aide d'une mêıne fonction, que l'on pourra appeler la fonction hydrogénique, laquelle devra jouer un rôle capital daus ces études : le résultat précédent paraît donc constituer un premier pas vers la solution des grands problèmes qui se posent en spectroscopie. 